\section{The first reported cases of $Q$ fever endocarditis in Thailand}

\author{
Orathai Pachirat, ${ }^{1}$ \\ Pierre-Edouard Fournier, ${ }^{2}$ \\ Burapha Pussadhamma, ${ }^{1}$ \\ Suthep Taksinachanekij, ${ }^{1}$ \\ Viraphong Lulitanond, ${ }^{1}$ \\ Henry C. Baggett, ${ }^{3}$ \\ Somsak Thamthitiwat, ${ }^{3}$ George Watt, ${ }^{3}$ \\ Didier Raoult, ${ }^{2}$ Susan A. Maloney ${ }^{3}$ \\ 'Departments of Medicine and \\ Cardiovascular Surgery, Faculty of \\ Medicine, Khon Kaen University, Khon \\ Kaen, Thailand; ' ${ }^{U}$ nite de Recherche sur \\ les Maladies Infectieuses et Tropicales \\ Emergentes, Faculte de Medicine, \\ University of the Mediterranean, \\ Marseille, France; ${ }^{3}$ International Emerging \\ Infections Program, Thailand MOPH-US \\ CDC Collaboration, Nonthaburi, Thailand
}

\section{Abstract}

We describe the first two reported cases of $Q$ fever endocarditis in Thailand. Both patients were male, had pre-existing heart valve damage and had contact with cattle. Heightened awareness of $Q$ fever could improve diagnosis and case management and stimulate efforts to identify risk factors and preventive measures.

\section{Introduction}

Blood culture-negative endocarditis (BCNE) is a term used in the cardiology literature to describe cases of infective endocarditis for which there is no bacterial growth in three independent blood samples cultured on standard aerobic media after seven days of incubation and subculturing. ${ }^{1}$ However, with specialized culture techniques, PCR, and serology, fastidious, slow growing bacteria can sometimes be identified in such cases. BCNE accounts for $2.5 \%-31.0 \%$ of all cases of endocarditis in industrialized countries, ${ }^{1}$ and for up to $76 \%$ of cases in the developing world. ${ }^{2}$ In Khon Kaen, Thailand, routine blood cultures identify a causative microorganism in only $38 \%$ of infective endocarditis patients, and BCNE is associated with a mortality of $37 \%{ }^{3}$

A collaborative study to examine the causes and risk factors for BCNE in Khon Kaen was begun in February 2010, with objectives to improve clinical outcomes and identify potential preventive interventions. Blood and cardiac tissue specimens collected during hospi- talization and heart valve replacement surgery from adult patients with suspected infective endocarditis were sent to the Emerging Tropical Infections Research Unit, Marseille, France and to the US CDC's Division of Vector Borne Diseases in Fort Collins, Colorado, for testing for bacterial pathogens known or suspected to cause BCNE. This collaboration led to the identification of the first two confirmed cases of $Q$ fever endocarditis in Thailand.

\section{Case Report \#1}

A 52-year old male rice and cattle farmer presented to hospital with a history of recurrent episodes of congestive heart failure during the previous two months. On admission, he was afebrile and had signs of severe aortic regurgitation. A transthoracic echocardiogram demonstrated severe aortic as well as mitral valve regurgitation. Six sets of blood cultures were negative. The patient underwent aortic valve replacement, and an aortic root abscess and perforation of the right coronary cusp were found intraoperatively. Serology was diagnostic for chronic $Q$ fever, with a serum IgG antibody titer of 1:25,600 to phase 1 antigens of Coxiella burnetii, the causative agent of $Q$ fever. IgG titers greater than 1:800 to phase $1 C$. burnetii are considered diagnostic of chronic $Q$ fever. ${ }^{4}$ The resected aortic valve tested positive by $16 \mathrm{~S}$ rRNA PCR for $C$. burnetii. Immunohistochemical staining demonstrated the causative organism within a background of chronic granulomatous inflammation (Figure 1).

\section{Case Report \#2}

A 42-year old male shoe salesman was admitted to hospital for aortic valve replacement. Past medical history was notable for chronic aortic valvular disease of 8 years duration. During the year prior to admission, the patient had experienced several episodes of congestive heart failure without fever. Three months prior to admission, the patient was referred to hospital because of severe aortic stenosis, aortic regurgitation and congestive heart failure and was treated for heart failure as an outpatient while awaiting surgery. On admission the patient was afebrile and had no stigmata of infective endocarditis. A transthoracic echocardiogram demonstrated no vegetative lesions. The patient underwent aortic valve replacement because of congestive heart failure but intraoperatively the aortic valve was noted to be badly damaged with multiple perforations, so the patient was referred for evaluation of possible infective endocarditis. The resected aortic valve was not tested by either
Correspondence: George Watt, IEIP, DDC 7 Building, 3rd Floor, MOPH Soi 4, Nonthaburi 11000 , Thailand.

Tel. +66.89 .927 .9928 . Fax: +66.2580 .0911 .

E-mail: georgew@th.cdc.gov

Key words: Coxiella burnetii, endocarditis, Q fever, Thailand.

Acknowledgements. we are grateful for the help of the Study Coordinator, Ms. Nongrak Ud-ai, for help with all phases of this project. Mr. Pongpun Sawatwong provided invaluable assistance with the logistics of obtaining and shipping specimens. We appreciate the help provided by Hubert Lepidi, Christopher Paddock and Pamela Greer with immunohistochemistry. Leonard Peruski, Nord Zeidner and Michael Kosoy were instrumental in planning the laboratory aspects of this investigation.

Contributions: all co-authors made substantial contributions to conception and design, acquisition of data, or analysis and interpretation of data. All co-authors contributed to the drafting of the article or to revising it critically for important intellectual content.

Conflict of interest: no potential conflicts of interest were identified.

Received for publication: 3 November 2011.

Revision received: 13 December 2012.

Accepted for publication: 27 December 2011.

This work is licensed under a Creative Commons Attribution NonCommercial 3.0 License (CC BYNC 3.0).

(C) Copyright O. Pachirat et al., 2012

Licensee PAGEPress, Italy

Infectious Disease Reports 2012; 4:e7

doi:10.4081/idr.2012.e7

PCR or immunohistochemistry for $C$. burnetii. After blood cultures were obtained, the patient was placed on broad-spectrum antibiotic therapy. Blood cultures were negative but serology was diagnostic for chronic $Q$ fever, with a serum anti-C. burnetii IgG1 antibody titer of $1: 6,400$. Although the patient did not raise cattle, several neighbors raised livestock, and exposure to these cattle is suspected.

\section{Discussion}

We describe the first two reported cases of $Q$ fever endocarditis in Thailand. C. burnetii, the causative agent, is most commonly acquired by inhalation of infectious aerosol particles from parturient or slaughtered ruminants. ${ }^{5}$ The exact mode of infection in our cases is unknown, but one patient owned cattle and the other is presumed to have had contact with a neighbor's cattle. 
These two cases illustrate several features characteristic of $Q$ fever endocarditis. Men account for the majority of cases ${ }^{6}{ }^{\text {and our }}$ patients were male. The clinical course is chronic and patients often lack the typical features of subacute, infective endocarditis. The majority of cases present with congestive heart failure due to severe valvular damage, while fever is often absent or low grade, as in our cases. ${ }^{6}$ As with Case 2, echocardiography is of limited value because vegetations are either
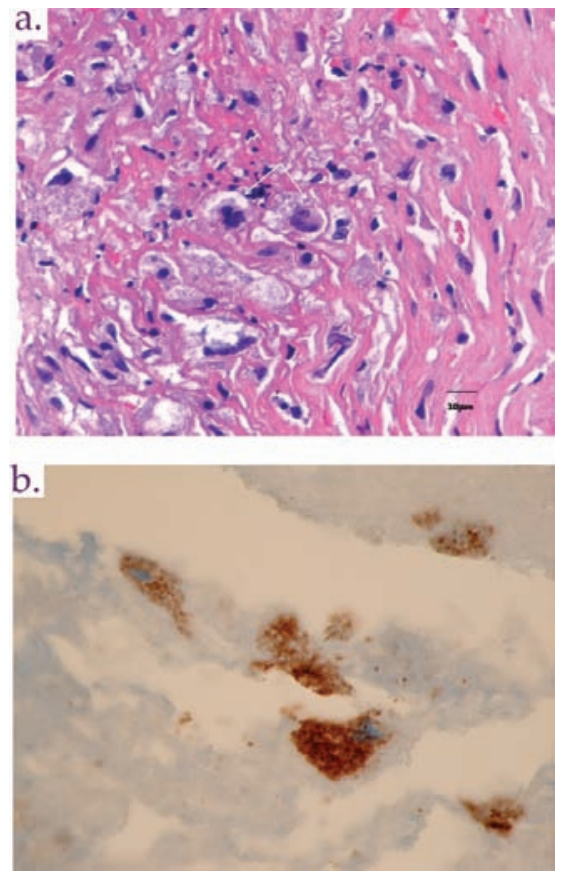

Figure 1. Resected aortic valve tissue from the patient described in case 1: A) an area of fibrin and calcification associated with active, suppurative inflammation and $B$ ) immunohistochemical localization of Coxiella burnetii antigens predominantly within macrophages. very small or are absent. ${ }^{6}$ Our patients had preexisting native valvular heart disease, probably rheumatic in origin. Rheumatic heart disease is associated with low socio-economic status and our study hospital serves Northeast Thailand, the poorest region of the country.

$Q$ fever is a common cause of BCNE worldwide. ${ }^{78}$ Without antibiotic therapy, $C$. burnetii endocarditis is usually fatal. ${ }^{9}$ It is therefore recommended that all patients with BCNE should have $Q$ fever excluded, although $Q$ fever diagnostic capability is often lacking in the developing world. ${ }^{4}$ Whenever possible, the diagnosis of $Q$ fever should be considered in the context of a comprehensive diagnostic strategy for BCNE. ${ }^{8}$

The results of the diagnostic testing in these cases were immediately conveyed to the treating cardiologist in Thailand (OP). The patients were contacted and placed on appropriate antibiotic therapy, because standard regimens for the treatment of BCNE would not be curative for $Q$ fever. Current treatment recommendations for $C$. burnetii endocarditis include doxycycline and hydroxychloroquine for 18 months for patients with infected native valves and 24 months for prosthetic valves. ${ }^{9}$ The duration of therapy is guided by monitoring serial anti-C. burnetii antibody titers. ${ }^{9}$

Previous publications have reported that acute $Q$ fever accounted for $1.3 \%$ of cases of acute, undifferentiated fever in Thailand but the true incidence in this country is unknown. ${ }^{10}$ More data on the incidence of $C$. burnetii infection in both humans and animals in Thailand are needed to understand the burden of disease in both populations. Increased awareness and earlier diagnosis of $Q$ fever endocarditis can significantly decrease associated morbidity and mortality. In addition to improving the treatment of individual cases, preventative measures could also be reviewed, as both human and animal $Q$ fever vaccines exist. ${ }^{5}$ Vaccination of patients with known valvular heart disease whose occupations place them at high risk for exposure to $C$. burnetii, such as those who raise livestock or work in slaughterhouses, might be one intervention to be considered to protect this high-risk group.

\section{References}

1. Brouqui P, Raoult D. Endocarditis due to rare and fastidious bacteria. Clin Microbiol Rev 2001;14:177-207.

2. Benslimani A, Fenollar F, Lepidi H, Raoult D. Bacterial zoonoses and infective endocarditis, Algeria. Emerg Infect Dis 2005;11: 216-24.

3. Pachirat 0, Chetchostisakd P, Klungboonkrong $\mathrm{V}$, et al. Infective endocarditis: prevalence, characteristics and mortality in Khon Kaen, 1990-1999. J Med Assoc Thai 2002;85:1-10.

4. Fournier PE, Marrie TJ, Raoult D. Diagnosis of Q Fever. J Clin Microbiol 1988;36:1823-34.

5. Parker NR, Barralet JH, Bell AM. Q fever. Lancet 2006;367:679-88.

6. Deyell MW, Chiu B, Ross DB, Alvarez N. Q fever endocarditis: a case report and review of the literature. Can J Cardiol 2006;22:781-5.

7. Houpikian P, Raoult D. Blood culture-negative endocarditis in a reference center. Medicine 2005;84:162-73.

8. Fournier PE, Thuny $\mathrm{F}$, Richet $\mathrm{H}$, et al. Comprehensive diagnostic strategy for blood culture-negative endocarditis : a prospective study of 819 new cases. Clin Infect Dis 2010;51:131-40.

9. Million M, Thuny F, Richet H, Raoult D. Long-term outcome of $Q$ fever endocarditis: a 26-year personal survey. Lancet Infect Dis 2010;10:527-35.

10. Suputtamongkol Y, Rolain JM, Losuwanaruk K, et al. Q fever in Thailand. Emerg Infect Dis 2003;9:1186-8. 